\title{
Genetic testing for vascular Ehlers-Danlos syndrome and other variants with fragility of the middle arteries
}

\author{
Yeltay Rakhmanov ${ }^{1}$, Paolo Enrico Maltese ${ }^{1 \star}$, Alice Bruson ${ }^{1}$, Marco Castori ${ }^{2}$, Tommaso Beccari ${ }^{5}$, \\ Munis Dundar ${ }^{6}$ and Matteo Bertelli ${ }^{1,5}$
}

\begin{abstract}
Ehlers-Danlos syndrome (EDS) is an umbrella term for various inherited connective tissue disorders associated with mutations in genes involved in extracellular matrix formation. "The 2017 International Classification of Ehlers-Danlos Syndromes and related disorders" identifies 13 clinical types with mutations in 19 distinct genes. The present module focuses on forms with major vascular involvement: vascular EDS (vEDS) caused by heterozygous mutations in COL3A1, "vascular-like" EDS (vlEDS) caused by recurrent mutations in COL1A1, classical EDS with vascular fragility associated with heterozygous mutations in COL5A1, and kyphoscoliotic EDS associated with recessive variations in PLOD1 and FKBP14. The overall prevalence of EDS is estimated between 1/10,000 and 1/25,000 and vEDS accounts for about 5 to $10 \%$ of all EDS cases. This Utility Gene Test was prepared on the basis of an analysis of the literature and existing diagnostic protocols. Molecular testing is useful for diagnosis confirmation, as well as differential diagnosis, appropriate genetic counselling and access to clinical trials.
\end{abstract}

Keywords: Kyphoscoliotic Ehlers-Danlos syndrome, vascular Ehlers-Danlos syndrome, COL1A1, COL3A1, COL5A1, FKBP14, PLOD1, EBTNA UTILITY GENE TEST

'MAGI's Lab, Rovereto, Italy

${ }^{2}$ Division of Medical Genetics, IRCCS-Casa Sollievo della Sofferenza, San Giovanni Rotondo (FG), Italy

${ }^{3}$ Department of Pharmaceutical Sciences, University of Perugia, Perugia, Italy

${ }^{4}$ Department of Medical Genetics, Erciyes University Medical School, Kayseri, Turkey

${ }^{5}$ MAGI Euregio, Bolzano, Italy

*Corresponding author: P. E. Maltese E-mail: paolo.maltese@assomagi.org

DOI: 10.2478/ebtj-2018-0034
Vascular Ehlers-Danlos syndrome and other variants with fragility of the middle arteries

(Other synonyms: classical Ehlers-Danlos syndrome with vascular fragility, vascular-like Ehlers-Danlos syndrome, kyphoscoliotic Ehlers-Danlos syndrome)

\section{General information about the disease}

Ehlers-Danlos syndrome (EDS) is of a group of inherited connective tissue disorders caused by mutations in genes involved in extracellular matrix formation. The mutations cause a loss of structural integrity in different organ systems. Different types of EDS exist, distinguishable on the basis of the gene(s) involved, clinical manifestations and prognosis. The present module considers forms of EDS with vascular involvement. Among the various forms of EDS, vascular EDS (vEDS; OMIM 130050) is the most common among variants with a major risk of middle artery ruptures and fragility (e.g. spontaneous ruptures) of hollow organs. In most patients vEDS is defined by typical facial features (e.g. large eyes, small chin, sunken cheeks, thin nose and lips, lobeless ears), acrogeria, translucent skin with evident subcutaneous vessels on the trunk and lower back, easy bruising and severe arterial, digestive and uterine complications. Complications are vascular and include arterial and venous abnormalities with arterial rupture (1-3). vEDS is always caused by heterozygous mutations in the COL $3 A 1$ gene.

Prevalence of EDS as a whole is estimated between $1 / 10,000$ and $1 / 25,000$, without significant ethnic variations. The "2017 International Classification of Ehlers-Danlos Syndromes and related disorders" identifies 13 clinical subtypes (4), among which 
vEDS probably accounts for about 5 to $10 \%$ of cases (5). The combination of two major criteria such as arterial rupture, intestinal rupture or uterine rupture during pregnancy and positive family history is strong diagnostic evidence of vEDS.

In cases who meet the clinical diagnostic criteria, genetic testing should be done to identify variations in COL $3 A 1$ in order to confirm the diagnosis $(2,4)$. Mutations in COL5A1 are normally associated with classical EDS (OMIM disease 130000) that is characterized by hyperextensible skin and joint laxity. However, some reports describe cases of classical EDS due to mutations in COL5A1 and potentially deadly middle artery fragility $(6,7)$. Rare families with classical EDS and vascular fragility are described with the recurrent mutations in COL1A1 (4). Vascular rupture has also been described in kyphoscoliotic EDS (OMIM disease 225400) caused by homozygous or compound heterozygous mutations in the PLOD1 and FKBP14 genes (8).

Differential diagnosis of vEDS should consider other forms of EDS, isolated arterial aneurysm, Loeys-Dietz syndromes, autosomal dominant polycystic kidney disease, Marfan syndrome, pseudoxanthoma elasticum and hereditary hemorrhagic telangiectasia (9).

vEDS regularly associates with mutations in COL3A1 (OMIM gene 120180). Heterozygous mutations in COL1A1 (OMIM gene 120150) and COL5A1 (OMIM gene 120215) may occasionally associate with similar phenotypes of middle artery ruptures/dissections/aneurysms. Recessive variants in PLOD1 (OMIM gene 153454) and FKBP14 cause the rarer kyphoscoliotic type.

Pathogenic variants may include missense, nonsense, splicing, small insertions, small deletions, small indels, gross insertions, gross deletions and complex rearrangements.

\section{Aims of the test}

- To determine the gene defect responsible for the disease;

- To confirm clinical diagnosis;

- To assess the recurrence risk and perform genetic counselling for at-risk/affected individuals.

\section{Test characteristics}

\section{Specialist centers/ Published Guidelines}

The test is listed in the Orphanet database and is offered by 23 accredited medical genetic laboratories in the EU, and in the GTR database, offered by 2 accredited medical genetic laboratories in the US.

Guidelines for clinical use of the test are described in Genetics Home Reference (ghr.nlm.nih.gov) and Gene Reviews (9).

\section{Test strategy}

In clear-cut phenotypes, targeted Sanger sequencing of $\mathrm{CO}$ $L 3 A 1$ may be considered a first tier approach for confirmation of the diagnosis. More commonly, a multi-gene next generation sequencing panel is used for the detection of nucleotide variations in coding exons and flanking introns of all the above genes.
Potentially causative variants and regions with low coverage are Sanger-sequenced. Sanger sequencing is also used for family segregation studies.

Multiplex Ligation Probe Amplification (MLPA) is used to detect duplications and deletions in the COL $3 A 1$ gene.

To perform molecular diagnosis, a single sample of biological material is normally sufficient. This may be $1 \mathrm{ml}$ peripheral blood in a sterile tube with $0.5 \mathrm{ml} \mathrm{K}$ EDTA or $1 \mathrm{ml}$ saliva in a sterile tube with $0.5 \mathrm{ml}$ ethanol $95 \%$. Sampling rarely has to be repeated.

Gene-disease associations and the interpretation of genetic variants are rapidly developing fields. It is therefore possible that the genes mentioned in this note may change as new scientific data is acquired. It is also possible that genetic variants today defined as of "unknown or uncertain significance" may acquire clinical importance.

\section{Genetic test results \\ Positive}

Identification of pathogenic variants in the above genes confirms the clinical diagnosis and is an indication for family studies.

A pathogenic variant is known to be causative for a given genetic disorder based on previous reports, or predicted to be causative based on loss of protein function or expected significant damage to proteins or protein/protein interactions. In this way it is possible to obtain a molecular diagnosis in new/other subjects, establish the risk of recurrence in family members and plan preventive and/or therapeutic measures.

\section{Inconclusive}

Detection of a variant of unknown or uncertain significance (VUS): a new variation without any evident pathogenic significance or a known variation with insufficient evidence (or with conflicting evidence) to indicate it is likely benign or likely pathogenic for a given genetic disorder. In these cases, it is advisable to extend testing to the patient's relatives to assess variant segregation and clarify its contribution. In some cases, it could be necessary to perform further examinations/tests or to do a clinical reassessment of pathological signs.

\section{Negative}

The absence of variations in the genomic regions investigated does not exclude a clinical diagnosis but suggests the possibility of:

- alterations that cannot be identified by sequencing, such as large rearrangements that cause loss (deletion) or gain (duplication) of extended gene fragments;

- sequence variations in gene regions not investigated by this test, such as regulatory regions (5' and $3^{\prime}$ UTR) and deep intronic regions;

- variations in other genes not investigated by the present test.

\section{Unexpected}

Unexpected results may emerge from the test, for example information regarding consanguinity, absence of family correla- 
tion or other genetically-based diseases.

\section{Risk for progeny}

If the identified pathogenic variant has autosomal dominant transmission, the probability that an affected carrier transmit the disease variant to his/her children is $50 \%$ in any pregnancy, irrespective of the sex of the child conceived.

In autosomal recessive mutations, both parents are usually healthy carriers. In this case, the probability of transmitting the disorder to the offspring is $25 \%$ in any pregnancy of the couple, irrespective of the sex of the child. An affected individual generates healthy carrier sons and daughters in all cases, except in pregnancies with a healthy carrier partner. In these cases, the risk of an affected son or daughter is $50 \%$.

\section{Limits of the test}

The test is limited by current scientific knowledge regarding the gene and disease.

Analytical sensitivity (proportion of positive tests when the genotype is truly present) and specificity (proportion of negative tests when the genotype is not present)

NGS Analytical sensitivity $>99.99 \%$, with a minimum coverage of 10X; Analytical specificity 99.99\%.

SANGER Analytical sensitivity $>99.99 \%$; Analytical specificity 99.99\%.

MLPA Analytical sensitivity >99.99\%; Analytical specificity 99.99\%.

\section{Clinical sensitivity (proportion of positive tests}

if the disease is present) and clinical specificity

(proportion of negative tests if the disease is not

present)

Clinical sensitivity: 95\% for vEDS (10).

Clinical specificity: nearly $100 \%$ (10).

\section{Prescription appropriateness}

The genetic test is appropriate when:

a) the patient meets the diagnostic criteria for EDS with vascular involvement;

b) the sensitivity of the test is greater than or equal to that of tests described in the literature.

\section{Clinical utility}

\section{Clinical management}

Confirmation of clinical diagnosis

Differential diagnosis

Couple risk assessment Yes

Availability of clinical trials can be checked on-line at https://clinicaltrials.gov/

\section{References}

1. Beridze N, Frishman WH. Vascular Ehlers-Danlos syndrome: pathophysiology, diagnosis, and prevention and treatment of its complications. Cardiol Rev 2012; 20(1): 4-7.

2. Callewaert B, Malfait F, Loeys B, De Paepe A. Ehlers-Danlos syndromes and Marfan syndrome. Best Pract Res Clin Rheumatol 2008; 22(1): 165-89.

3. Germain DP. Ehlers-Danlos syndrome type IV. Orphanet J Rare Dis 2007; 2: 32.

4. Malfait F, Francomano C, Byers P, Belmont J, Berglund B, Black J, Bloom L, Bowen JM, Brady AF, Burrows NP, Castori M, Cohen H, Colombi M, Demirdas S, De Backer J, De Paepe A, Fournel-Gigleux S, Frank M, Ghali N, Giunta C, Grahame R, Hakim A, Jeunemaitre X, Johnson D, Juul-Kristensen B, Kapferer-Seebacher I, Kazkaz H, Kosho T, Lavallee ME, Levy H, Mendoza-Londono R, Pepin M, Pope FM, Reinstein E, Robert L, Rohrbach M, Sanders L, Sobey GJ, Van Damme T, Vandersteen A, van Mourik C, Voermans N, Wheeldon $\mathrm{N}$, Zschocke J, Tinkle B. The 2017 international classification of the Ehlers-Danlos syndromes. Am J Med Genet C Semin Med Genet 2017; 175(1): 8-26.

5. Barabas AP. Vascular complications in the Ehlers-Danlos syndrome, with special reference to the „arterial type" or Sack's syndrome. J Cardiovasc Surg (Torino) 1972; 13(2): 160-167.

6. Monroe GR, Harakalova M, van der Crabben SN, Majoor-Krakauer $D$, Bertoli-Avella AM, Moll FL, Oranen BI, Dooijes D, Vink A, Knoers NV, Maugeri A, Pals G, Nijman IJ, van Haaften G, Baas AF. Familial Ehlers-Danlos syndrome with lethal arterial events caused by a mutation in COL5A1. Am J Med Genet A 2015; 167(6): 1196-1203.

7. Mehta S, Dhar SU, Birnbaum Y. Common iliac artery aneurysm and spontaneous dissection with contralateral iatrogenic common iliac artery dissection in classic ehlers-danlos syndrome. Int J Angiol 2012; 21(3): 167-170.

8. Rohrbach $M$, Vandersteen A, Yiş U, Serdaroglu G, Ataman E, Chopra M, Garcia S, Jones K, Kariminejad A, Kraenzlin M, Marcelis C, Baumgartner M, Giunta C. Phenotypic variability of the kyphoscoliotic type of Ehlers-Danlos syndrome (EDS VIA): clinical, molecular and biochemical delineation. Orphanet J Rare Dis 2011; 6: 46.

9. Pepin MG, Murray ML, Byers PH. Vascular Ehlers-Danlos Syndrome. In: Adam MP, Ardinger HH, Pagon RA, et al., editors. GeneReviews ${ }^{\oplus}$ (Internet) 2015; Seattle (WA)

10. Mayer K, Kennerknecht I, Steinmann B. Clinical utility gene card for: Ehlers-Danlos syndrome types I-VII and variants - update 2012. Eur J Hum Genet 2013; 21(1). 